\title{
Disseminate time-sensitive research faster
}

Previously published at www.cmaj.ca

I mpressive results of a large multicentre clinical trial are announced at a prominent international scientific meeting. The study's conclusions receive widespread media coverage, but the paper describing the details has not been published.

A government regulatory agency announces that a pattern of unexpected adverse reactions to a drug on the market has been observed in an ongoing clinical trial. The agency issues a public alert to health professionals. However, the trial is scheduled to continue for two years, and a detailed analysis of the findings is not yet public.

Public health agencies announce a policy change affecting distribution of and recommendations for receiving a vaccine, on the basis of findings from a new national study. Only the conclusions of the study are released while the study undergoes peer review at a medical journal. Soon after, a study from another country is published that appears to show contradictory findings.

These are real — and increasingly common - examples of mounting challenges posed by new research findings that are both very important and time sensitive. In an era of instant communication, traditional dissemination processes used by researchers, policy-makers, regulators and journals may prove inadequate for health professionals and the public, particularly during health emergencies or for reporting possible new risks of widely prescribed therapies.

A common element of the challenges is initial widespread reporting by the media, which usually conveys no more than a study's bottom line and often directs the public to seek advice from their health care providers. But when patients' questions arise from unpublished — and inaccessible — study results, practitioners are in an impossible position. Unable to appraise the research, clinicians are left with an uncertain foundation for making decisions about patient care and, at best, can only echo what has been said publicly by others.

The usual process of research dissemination - peer review and publication in scientific journals - aims to ensure the credibility and validity of science and enhances the clarity and effectiveness of scientific communication. True emergencies, or single studies whose results should immediately change practice, are uncommon. Health care practice most often changes slowly and incrementally by accumulation of evidence over time. Consequently, a process that emphasizes ample time for reflection and correction is usually most appropriate.

The challenge comes in recognizing and acting on the exceptional circumstances in which the usual approach is insufficient. Communication of important research findings that have immediate implications for public health can, and must, be improved. All stakeholders in the publication process need to develop the capacity to make the process work faster when speed is critical.

Authors must advocate for their work and disseminate their findings as effectively as possible. This means not only choosing the most appropriate medium that targets the appropriate audience, but also being proactive in contacting and negotiating with journal editors as early as possible.

Regulatory and public health agencies are responsible for acting quickly on new scientific information and making decisions to try to protect the population. But such decisions must be informed adequately. Such agencies must be equally quick to review new science, especially during health emergencies or when large amounts of new information are generated over short periods. They must also make enough information available to the practitioners who will implement their decisions to ensure that the basis for such decisions is clearly understood.

Medical journals such as CMAJ must continue to find ways to publish work faster. Online publication and mechanisms for fast-track review have made publication far more timely. However, thoughtful advance planning is required to ensure that these processes function well in an emergency.

When circumstances demand that the publication of research be fast-tracked, two principles are key. First, although the important steps of peer review and revision must be accelerated, none should be skipped for time's sake. Medical journals must not shortchange the process's quality and integrity, which are even more essential during health emergencies to ensure credibility. Second, all parties must ensure that research — and how it is being appraised — is made known with maximum transparency.

\section{Matthew B. Stanbrook MD PhD \\ Deputy Editor, Scientific \\ Paul C. Hébert MD MHSc \\ Editor-in-Chief \\ CMAJ \\ Editorial advisory team: Ken Flegel MDCM MSc, Noni MacDonald MD MSc and Joan Ramsay BA}

Competing interests: See www.cmaj.ca/misc/edboard.shtml

CMAJ 2009. DOI:10.1503/cmaj.092077 